\title{
Pros y contras percibidos \\ de la actividad física realizada \\ por adultos mayores
}

\author{
Perceived pros and cons of physical activity by older adults
}

MES. Ma. Rosalinda Medina Briones*, MES. Martha López Hernández*, MCE. Martha Lilia Zuñiga Vargas*,

LSHI Leticia Ibarra Mata**

*Profesor de Carrera G, ** Pasante de maestría

\section{Resumen}

INTRODUCCION: La práctica de actividad física regular es una de las prioridades en salud pública para incrementar el bienestar y la calidad de vida especialmente en la tercera edad. Esta práctica depende de varios factores, entre otros las experiencias previas de la conducta y la valoración de las ventajas y desventajas que tiene su realización.

Propósito: Conocer los pros (ventajas) y Contras (desventajas) percibidos del ejercicio físico en adultos mayores.

METODOLOGÍA: En este estudio se utilizó el balance decisional (Prochaska y Di Clemente,1997). Se aplicó la aproximación cualitativa con muestreo teórico y análisis de contenido en una muestra de 15 participantes.

Resultados: Estos mostraron tres categorías: Pros de la actividad física, construida a partir de las subcategorías: mejorar la salud física y emocional e incrementar las relaciones interpersonales. La segunda categoría: Contras, se construyó a partir de las subcategorías ambientales, ocupaciones, enfermedades y ser cuidadores de otras personas y una tercer categoría identificada se construyo con las razones para realizar la actividad física.

CONCLUSIONES: Los pros y contras señalados por los participantes pueden ser la calve para el diseño de estrategias de programas de salud encaminados a incluir la actividad física en el adulto mayor.

Palabras claves: Percepción de Pros y Contras, Actividad Física, Adulto Mayor

\section{Abstract}

Introduction: The practice of regular physical activity is one of the priorities in public Health to increase the well-being and the quality of life especially in the third age. This practice depends on several factors, among others the previous experiences of the conduct and evaluation of the advantages and disadvantages upon achievement.

Purpose: Acknowledge pros and cons detected on physical exercise on older adults.

Methodology: In this study the decisional balance (Prochaska and Di Clemente,1997) was used. The qualitive approach was applied by theoretical sampling and analysis of content sampling 15 participants.

Results: They showed this categories: pros of the physi- cal activity, built from the subcategories: to improve the physi$\mathrm{cal}$ and emotional health and to increase the

interpersonal relations. The second category, cons was formed from environmental

Subcategories: Occupations, diseases and to carers of others persons and a third

Identified category was the reasons of physical activity.

Conclusions: The pros and cons distinguished by the participants can be the key for the strategic health programs design aimed to include physical activity on major adults.

Key words: Pros \& Cons, perception, physical actrivity, Older Adults. 


\section{INTRODUCCIÓN}

El envejecimiento de la población constituye uno de los acontecimientos sociales y demográficos relevantes de las últimas décadas. La creciente población de adultos mayores debe ser integrada al desarrollo y la modernidad lo que depende en parte importante del Estado, pero también de los propios adultos mayores y los individuos que se aproximan a esta etapa de la vida'1. En México existe una tendencia al incremento de la población de adultos mayores la cual tiene entre sus explicaciones el mejoramiento de las condiciones socioeconómicas de la población, aumento en la esperanza de vida y disminución de la mortalidad. En México la vejez dejó de ser la experiencia de unos cuantos para convertirse en la cotidianidad de millones. Hoy la población mayor de 65 años asciende a poco más de cinco millones de personas. En 2050 los adultos mayores serán 32 millones y representarán 24.6\% de la población. A diferencia de lo que ocurre con otros grupos de edad, la población de 65 años de edad y más está creciendo a una tasa anual de $2 \%$ y, de manera sorprendente, el ritmo de crecimiento de los mayores de 85 años se ubica entre 3 y $5 \%{ }^{2}$

Uno de los factores vitales para el incremento en la esperanza de vida, por ende de la población de adultos mayores, corresponde al estilo de vida en donde la actividad física es un elemento que permite que se sientan mejor, adquiriendo en su cuerpo una resistencia superior ante las distintas agresiones que el paso de los años provocan. Los adultos que conservan una vida activa llegan a edades mayores con mejor predisposición al trabajo y menor dependencia de aquellos que lo rodean. ${ }^{3,4}$

La actividad física se refiere a todo movimiento corporal que implique un gasto energético y que se encuentra en su mayor parte bajo control voluntario. ${ }^{5}$ El ejercicio físico es un componente de la actividad física y se define como movimientos corporales planificados, estructurados y repetitivos que se realizan con o sin fines de acondicionamiento físico. ${ }^{6}$

Este tipo de movimiento corporal es útil para mantener el estado normal de los músculos y articulaciones; prevenir la perdida de calcio en los huesos (osteoporosis) aumentar la capacidad de los sistemas respiratorio y cardiovascular; evitar el daño de las arterias y mejorar la coagulación sanguínea. Además, es muy importante para lograr una actitud emocional positiva de confianza en sí mismo. ${ }^{3}$

La práctica del ejercicio físico regular es una de las prioridades en salud pública como forma de prevención de enfermedades crónico degenerativas especialmente en la tercera edad. La decisión de realizar un cambio en el estilo de vida o bien el inicio de una acción o conducta saludable como la actividad física depende de varios factores entre ellos, el apoyo social que perciben de su familia, experiencias previas de la conducta y la valoración que realiza de las ventajas o desventajas que tiene su realización.

Prochaska y Di Clemente ${ }^{7}$ (1997) elaboraron el Modelo Transteorico de Etapas de Cambio donde el constructo considera el peso relativo de los Pros y Contras para iniciar y mantener un proceso de cambio de conducta por los individuos o para tomar una decisión que guíe una conducta.

Además el concepto Balance Decisional refleja el valor relativo que le otorga un individuo a los pros (creencias, percepciones o consecuencias positivas sobre los beneficios de desarrollar una conducta) y contras (creencias o percepciones de los costos o consecuencias negativas del desarrollo de una conducta) para iniciar o mantener una conducta como es la actividad física abordada en este estudio. Los autores del Modelo Transteorico han señalado que cuando son percibidos en mayor proporción los pros y en menor los contras la acción tiende a iniciarse y mantenerse. Se han realizado estudios desde esta perspectiva ante la actividad física encontrando que los adultos mayores perciben que ésta les ayuda a incrementar su calidad de vida ya que desarrollan resistencia a la fatiga, se disminuye su ansiedad o depresión, favorece la conciliación del sueño, mejora las relaciones con amigos y familia, ofrece mayor energía para las actividades diarias, facilita el movimiento de las articulaciones y contribuye a la pérdida de peso cuando éste es necesario. Entre las limitaciones que refieren los adultos mayores para realizar la actividad física se encuentran problemas de salud, el cuidado de los nietos, padres o esposos, responsabilidades del hogar y la falta de espacios para realizar alguna actividad física. ${ }^{8,9}$

Actualmente se ha utilizado el Modelo Transteorico en diversos estudios de la actividad física como el de Autoeficacia y Escenarios de Cambio, realizado en estudiantes irlandeses, donde determinaron que la etapa de cambio puede explicar las diferencias de actividad por sexo ${ }^{10}$. Otro estudio en población alemana encontró diferencia entre la disposición al cambio, los pros y contras percibidos y la autoeficacia, encontrando que los pros percibidos la promueven y los contras la limitan ${ }^{11}$. 
La actividad física como conducta promotora de salud en los adultos mayores es una meta del cuidado de enfermería que incrementa la salud y bienestar en este grupo de población ${ }^{12}$, en este sentido la Universidad Autónoma de Tamaulipas promueve la extensión y vinculación con la sociedad tamaulipeca a través de la estrategia de crear los Centros Multidisciplinarios para la Formación Integral, proporcionando una especial atención al cuidado de los adultos mayores en los denominados clubes de la edad de oro. En estos se realizan múltiples actividades que promueven la actividad física como el baile, ejercicio cardiovascular y Tai Chi, sin embargo se ha observado por los autores del estudio que la asistencia de los adultos mayores varia en ciertos períodos del año, por lo que resulta importante conocer cuáles son sus motivos.

Por lo anterior el objetivo del presente estudio fue Conocer los pros y contras percibidos para la práctica de la actividad física en adultos mayores que asisten al Centro Multidisciplinario de la UAT de Nuevo Laredo con la finalidad de que en un futuro se establezcan programas de promoción de la salud enfocados al reforzamiento de esta conducta.

\section{METODOLOGÍA}

Se utilizo la aproximación cualitativa con la perspectiva del interaccionismo simbólico ya que éste atribuye una importancia primordial a los significados sociales que las personas le asignan al mundo que los rodea y permite conocer los pros (beneficios) y contras (barreras) de la actividad física que los informantes han construido a través de los significados del valor que tiene la actividad física en sus vidas y en razón de ellos desarrollan la conducta, la cual se refuerza en la interacción con los demás . 13,14 Se hizo con muestreo teórico y la técnica de bola de nieve, colaboraron 15 adultos (7 hombres y 8 mujeres) mayores de 60 años, del grupo de la "Edad de Oro" del Centro Multidisciplinario que acudieron a una invitación hecha en cartelón donde se estableció lugar y hora para la entrevista. El número de participantes se considero suficiente debido a la saturación de la información. Las entrevistas fueron semi-estructuradas a partir de una pregunta central ¿qué ventajas (pros) y desventajas (contras) identifica usted en la realización de actividad física en el Centro Multidisciplinario? Las entrevistas fueron audio grabadas previo consentimiento de los informantes y se

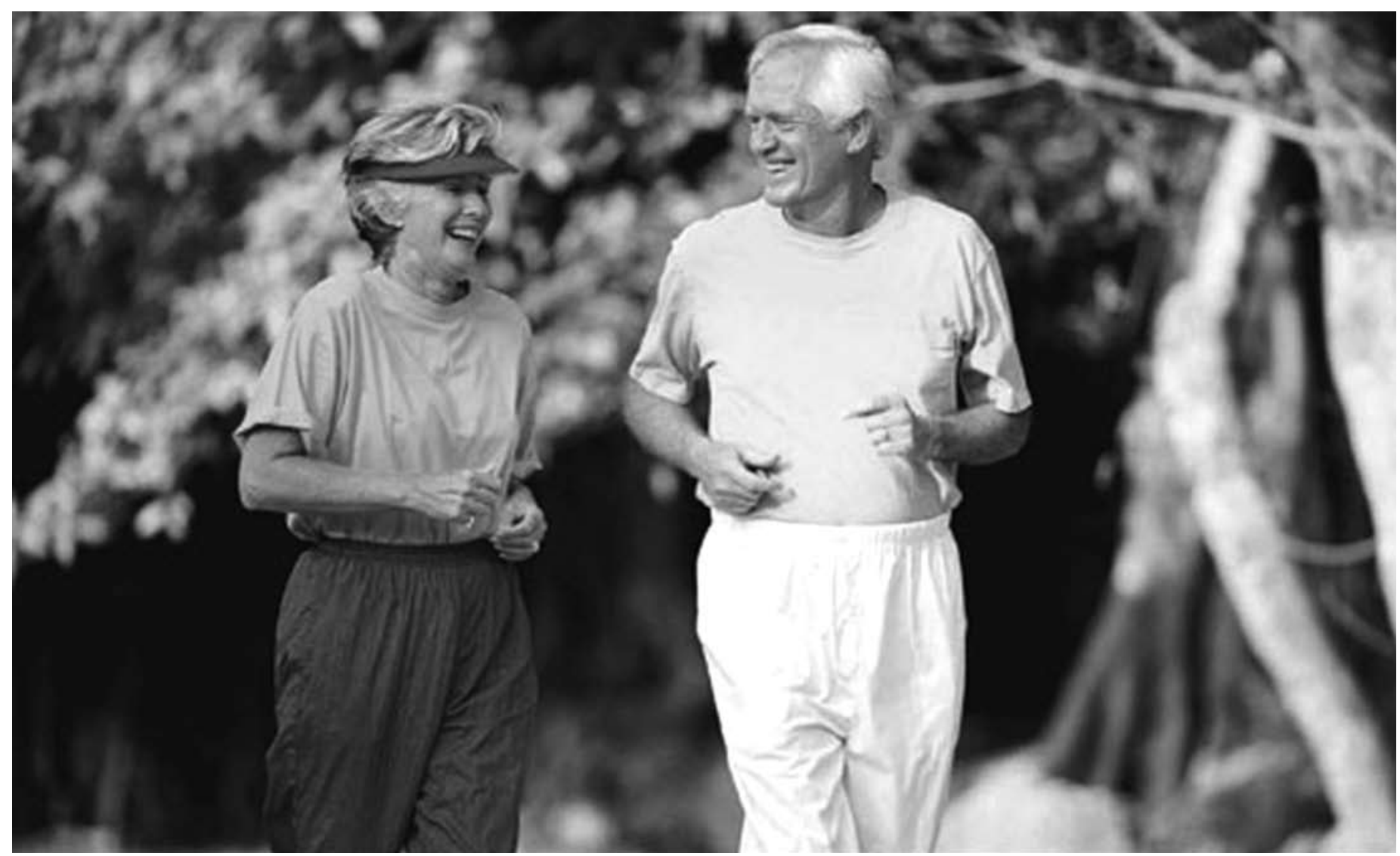


transcribieron en formato Word. El análisis de datos se realizó siguiendo el proceso de encontrar sentido al dato cualitativo por medio del Análisis de Contenido ${ }^{15}$, lo que implico un microanálisis del texto línea por línea, se obtuvieron códigos vivos que se procesaron y redujeron a través de la codificación abierta, axial y selectiva para definir los códigos vivos representativos en cada categoría y subcategoría encontrada.

El estudio se apego a lo dispuesto por el Reglamento de la Ley General de Salud en materia de investigación ${ }^{16}$, se obtuvo el consentimiento informado por escrito de todos los entrevistados, garantizándoles el anonimato y la confidencialidad de la información.

\section{RESULTADOS Y DISCUSIÓN}

Se identificaron las dos categorías centrales que fueron derivadas del Modelo Transteorico de Prochaska y Di Clemente, adicionalmente se identificó una tercera que los autores del estudio señalaron como razones o motivaciones de la conducta.

Los participantes relatan en el cuadro 1, que la actividad física les ha incrementado su fuerza, flexibilidad y equilibrio, además de reducir el estrés, estos hallazgos se han encontrado además tanto en mujeres adultas como en varones. ${ }^{8}$ Un aspecto importante que se desprende de la actividad física es lo señalado por los participantes cuando narran que esta les incrementa la convivencia, la alegría de vivir, compartir tiempo e intereses con otras personas, se sienten contentos y con mente positiva ${ }^{17,18}$. Estas emociones o afecto que se desprenden de la conducta son factores reforzadores que permiten que ésta se mantenga en el tiempo ${ }^{19}$.

En el cuadro 2 se narran los contras o desventajas percibidas que limitan la actividad física, destacando los factores ambientales como el clima o las ocupaciones de tipo familiar ${ }^{9,20}$.

Un aspecto que forma parte de la cultura de la frontera norte del país es el transito constante hacia los Estados Unidos con lo cual ocurre el proceso de aculturación, entendido este como la adquisición de estilos de vida, valores, el consumo de bienes y servicios (las compras) y las prioridades familiares. Como resultado de este contacto sistemático con otra cultura, estos factores pueden ser limitantes o facilitadores de la actividad física ${ }^{21,22}$, en este estudio se observa como una limitante de la actividad física.

Otra subcategoría identificada fue padecer alguna enfermedad como la "gripa o resfriado" y complicaciones derivadas de enfermedades crónico-degenerativas, convirtiéndose en obstáculos para el desarrollo de la conducta según Prochaska y Di Clemente. Otro aspecto que parte de la cultura mexicana, es que los abuelos cuiden de los nietos o bien los hijos mayores de sus padres de edad mucho más avanzada, ${ }^{23}$ ocupación que de tener que realizarla se constituye en una limitante para que los adultos mayores acudan a realizar la actividad física,

Las motivaciones señaladas en el cuadro 3 están relacionadas con evitar la vida sedentaria que de alguna forma se relaciona con los pros percibidos donde los adultos mayores reconocen y han experimentado el hecho de que la actividad física mejora su condición de salud, sus capacidades y resistencia física.

Es importante señalar que los informantes relatan con frecuencia que entre las motivaciones para realizar la actividad física, está la de ver el mundo de otra manera, tener más amistades y convivir con otras personas, no quedarse en casa, salir de viaje, festejar los cumpleaños y estar felices, en otras palabras se ésta hablando de las necesidades de ellos respecto a evitar la soledad, búsqueda de emociones positivas, ser reconocidos como parte de un grupo. Lo anterior se desprende de la etapa de vida en que se encuentran de retiro y jubilación donde han tenido múltiples perdidas como empleo, amigos, los hijos que forman nuevas familias, perdidas de compañeros de trabajo y responsabilidades que generan un estatus y que son afrontadas mediante la actividad física.

\section{CONCLUSIONES}

Fue factible la aplicación empírica del constructo balance decisional con metodología cualitativa según lo reportado en el presente estudio.

La categoría de pros de la actividad física se construyó a partir de las subcategorías de mejorar la salud física y emocional e incrementar las relaciones interpersonales. La segunda categoría denominada contras se construyó a partir de las subcategorías ambientales y ocupaciones y de enfermedades y cuidadores de otras personas.

Una tercera categoría fue la de razones de la actividad física que reconocen los adultos mayores entre estas se encuentran las subcategorías, seguir con un ritmo de vida, gusto y placer y viajar y festejar.

Los pros y contras señalados por los participantes pueden ser la calve para el diseño de estrategias de programas de salud encaminados a incluir y promover fuertemente la actividad física en el adulto mayor. 


\section{AGRADECIMIENTOS}

Los autores agradecen a los adultos mayores participantes del estudio su tiempo y la información proporcionada para el presente estudio.

\section{REFERENCIAS BIBLIOGRÁFICAS}

1 Sepúlveda AD. Cuidado y Autocuidado de Salud del Adulto Mayor. Activity Intervention. Annals of Behavioral Medicine. (consultado 30/03/2009 on line) 2004;20:174-180 www.gerontologia.uchile.cl/docs/cuidado.htm

2 Secretaría de Salud. Programa Nacional de Salud 20072012. Por un México Sano: construyendo alianzas para una mejor salud. Primera edición. México, D. F.

3 Peidro R. "Ejercicio físico para la salud y la calidad de vida". 2002 (consultado 21/04/2008 on line) Disponible en: www.bago.com/Cardired/Ejercicio_salud/Ejercicio_salud.asp

4 Soltero SG, Salazar BC. Autoesquemas de Ejercicio Físico Reportados por Adultos Mayores. Monterrey, México. Invest Educ enfer 2006; (24)2:86-89

5 Hernández B, Gortmaker S, Laird N, Colditz G, Parra-Cabrera S, Peterson K. Validez y Reproducibilidad de un Cuestionario de Actividad e Inactividad Física para Escolares de La Ciudad de México. Salud Pública de México. 2000; 42 (4): 315-323

6 Keysor JJ. Does Late-Life Physical Activity or Exercise Prevent or Minimize Disablement? A Critical Review of the Scientific Evidence. American Journal of Preventive Medicine. 2003; 25: 129-136

7 Prochaska JO \& Di Clemente CC. The Transteorical Model and Stages of Change. Health Behavior and Health Education. $2^{a}$ Ed. 1997. 60-66 pp.

8 Cardinal BJ, Sachs M L. Effects of Mail-Mediated, StageMatched Exercise Behavior Change Strategies on Female Adults' Leisure-Time Exercise Behavior. Journal of Sports Medicine and Physical Fitness. 1996; 36:100-107.

9 Marcus $\mathrm{BH}$ y Col. Efficacy of an Individualized, MotivationaIly Tailored Physical Activity Intervention. Anals of Behavioral Medicine.1998, 20:174-180.

10 Patterson E y Col. Self-efficacy, Stages of Change and Physical Activity in Irish College Students. Journal of Public Health. 2006; 14: 81-86

11 Smeets T \& Vries H. Misconceptions about Physical Activity. (Abstract). Trabajo presentado en el 8th International Congress of Behavioral Medicine. Integrating Social and Behavioral Sciences with Medicine and Public Health. Maínz. Germany. 2004
12 Robbins, Lorraine B.; Pender, Nola J.; Conn, Vicki S.etal Physical Activity Research in Nursing. Journal of Nursing Scholarship. Fourth Quarter 2001.33(4):315-321.

13 Denzin N \& Lincoln Y. The Landscape of Cualitative Research. Theories and Issues. Londres: Sage Publication. Tomo1. 1998. $407-430 \mathrm{pp}$.

14 Taylor SJ, Bogda R. Introducción a los Métodos Cualitativos de investigación. Editorial Paidos, $3^{a}$ Ed. España. 2000. 10-23 pp.

15 Coffey A \& Atkinson P. Encontrar el Sentido de los Datos Cualitativos. Estrategias Complementarias de Investigación. Ed. Universidad de Antioquia.2004. 31-63 pp

16 Secretaria de Salud. Reglamento de la Ley General en Materia de Investigación en Salud. México. 1997

17 Barrios DR, Borges MR, Cardoso PL. Benefícios percibidos por adultos mayores incorporados al ejercicio. Rev Cubana Méd Gen Inter. 2003; 19(2) (consultado 28/07/2008 on line) Disponible en: www.Infomed.Sid.cu

18 Salinas CJ, etal. Actividad Física Integral con Adultos Mayores Chile: Resultados de Un Programa Piloto. Rev. Chil. Nutr. 2005; 32(3):215-224 (consultado 09/05/2009 on line) http://www.scielo.cl/scielo.php?script=sci_arttext\& pid $=$ S071775182005000300006

19 Finfforff M J etal. Does the Transtheretical Model (TTM) Explain Exercise Behaivor in a Community - Based Sample of Older Women? Rev. Journal and Health. 2007; 19(6): $985-1003$

20 De la Calle PM, Barriopedro MM, Aznar LS. Niveles de adherencia en postinfartados. 2004. (Consultado 10/07/2008 on line). Universidad Europea de Madrid. Universidad de Castilla La Mancha. http:www.unex.es/eweb/ cienciadeporte/congreso/04\%20val/pdf/c91.pdf,

21. Acosta GV y Cols. Evaluación de la conducta alimentaria de riesgo. Estudio transcultural entre adolescentes de España y México. International Journal of Psychology and Psycological Therapy. 2005; 5(3): 223-232

22 Rojas TJ, Zubizarreta E M. Reflexiones del estilo de vida y vigencia del autocuidado en la atención primaria de salud. Rev. Cubana Enfermer. 2007; 23(1)

23 Ribeiro,Rondeau,Hernández. (2002). La Familia en América del Norte: Evolución, Problemática y Política. Trillas. Mexico,2002. $368 \mathrm{pp}$.

\section{DIRECCIÓN PARA CORRESPONDENCIA}

Ma. Rosalinda Medina Briones: rmedina@uat.edu.mx 


\section{Cuadro 1 Pros Percibidos de la Actividad Física}

\begin{tabular}{|c|c|}
\hline $\begin{array}{l}\text { Categoría(Pros )Son creencias, percepciones } \\
\text { o consecuencias positivas sobre beneficios } \\
\text { de realizar la conducta }\end{array}$ & $\begin{array}{l}\text { Códigos Vivos son las expresiones de adultos mayores construidas s } \\
\text { obre el significado de pros }\end{array}$ \\
\hline \multicolumn{2}{|l|}{ Subcategorías } \\
\hline Mejorar la salud física y emocional & $\begin{array}{l}\text { Me ha ayudado lo que es el ejercicio de la caminadora, el baile y ahora el taichí a estar } \\
\text { mas fuerte. (E02) (E05) (E07) } \\
\text { Porque me siento fuerte, me siento joven o sea, creo que los limites permitidos, ver- } \\
\text { dad? (E03) (E09) } \\
\text { Me siento mas tranquila, me libero del estrés, bailar me entusiasma y me da margen a } \\
\text { que yo también lo puedo realizar (E11) }\end{array}$ \\
\hline Incrementar las relaciones interpersonales & $\begin{array}{l}\text { Tienes muchas amistades, mucha convivencia, muchos viajes (E02) } \\
\text { Pues bastante porque es tanto físico, mental y social me relaciono con mis compañeras } \\
\text { (E05)(E06)(E09) } \\
\text { Nuestra forma de vivir, de celebrar cumpleaños, a estar contentos, a estar alegres, a } \\
\text { estar felices (E11) (E15) }\end{array}$ \\
\hline
\end{tabular}

\section{Cuadro 2 Contras Percibidos de la Actividad Física}

Categoría(Contras)Son creencias o percepciones de costos del desarrollo de la conducta

\section{Subcategorías}

Ambientales y de Ocupaciones

Enfermedades y cuidadores

de otras personas
Códigos Vivos son las expresiones de adultos mayores

construidas sobre el significado de contras

Muchas veces no lo puedes realizar porque llueve que porque

hace frío (E02 Tengo un compromiso no voy a venir (E01)

Si quiero ir porque debo hacer vueltas a Laredo Texas, si tengo ganas de ir, ya no estoy sujeta a tiempos pero eso me hace faltar al ejercicio (E01,E06)

He faltado a veces por los viajes, porque viajamos en familia (E02,E03)

Se enferma uno de la gripa o se me complica la diabetes (E02,E04,E10)

Yo me llevé siete años imposibilitada de andar en silla de ruedas, andador, bastón y mijo me inició aquí y ahora (E04)

No, no a excepción de una pequeña molestia en la rodilla $(E 05, E 09)$

En ocasiones falto porque, tengo que cuidar a mis nietos, no viven aquí, llegan de viaje de paseo a la casa y pues regularmente dejo de venir, cuido a mi esposo, mis padres imposibilitados(E02,E09,E13,E14)

\section{Cuadro 3 Razones de la Actividad Física}

Categoría (razones) son las motivaciones o incentivos de los adultos mayores para realizar actividad física

Seguir con un ritmo de vida

Gusto o placer

Viajar y festejar
Códigos Vivos son las expresiones de adultos mayores construidas sobre las razones que justifican la conducta

La vida sedentaria no es buena para nosotros (E01,E02)

Sentirse uno mejor, mirar al mundo de otra manera (E11,E12)

A mi siempre me ha gustado el baile y el taichí fue extra (E01) (E13)

Estoy bien contenta de estar aquí (E04,E15)

Tienes muchas amistades, mucha convivencia, mucha los viajes que hemos hecho (E02) (E06) (E12)

hemos salido a, a concursar a Tampico, a Matamoros, a Reynosa (E09,E07)

nuestra forma de vivir, de celebrar cumpleaños, a estar contentos, a estar alegres, a estar felices $(E 15, E 03)$ 